\title{
ENHANCEMENT OF TRANSMISSION RANGE ASSIGNMENT FOR CLUSTERED WIRELESS SENSOR NETWORKS
}

\author{
M. Salamah and A. A. Hussein \\ Computer Engineering Department, Eastern Mediterranean University, TRNC, Mersin \\ 10, TURKEY
}

\begin{abstract}
Transmitter range assignment in clustered wireless networks is the bottleneck of the balance between energy conservation and the connectivity to deliver data to the sink or gateway node. The aim of this research is to optimize the energy consumption through reducing the transmission ranges of the nodes, while maintaining high probability to have end-to-end connectivity to the network's data sink. We modified the approach given in [1] to achieve more than 25\% power saving through reducing cluster head (CH) transmission range of the backbone nodes in a multihop wireless sensor network with ensuring at least 95\% end-to-end connectivity probability.
\end{abstract}

\section{KEYWORDS}

Wireless sensor networks, wireless ad hoc networks, clustering, transmission range, connectivity.

\section{INTRODUCTION}

In the literature follow, clustering mechanism in wireless networks means dividing network's nodes into groups which are called clusters. Each cluster has a single cluster head $(\mathrm{CH})$ which collects and summarizes data flow from ordinary cluster's nodes and forwards it to the Gateway nodes which are shared between two or more clusters.

The main advantages of clustering in wireless sensor networks (WSN) are: reducing traffic volume of data flows by forming the $\mathrm{CH}$-backbone; making the network topology more simple; and alleviating overhead, collision, interference and traffic congestion [1][2]. Ordinary nodes in clusters elect their $\mathrm{CHs}$ via $\mathrm{CH}$ candidacy announcements performed by each node according to a probability scale which is computed by individual nodes and it considers the effect of hop distances to the sink on the relative traffic loads at different locations of the network [3-5].

In clustering protocols, the main aim is the successful delivery of network data to the gateway. However, there are two main related concepts. If the transmission range of $\mathrm{CH}$-to- $\mathrm{CH}$ is too short (not long enough), it will drain low transmission power, but leads to network partitioning in which some $\mathrm{CHs}$ cannot communicate, and hence causes failure of data delivery process to the gateway [5]. On the other hand, if the transmission range of $\mathrm{CH}$-to- $\mathrm{CH}$ is too long (not short enough), it will ensure the successful delivery of network data to the gateway, but requires high transmission power and difficult modulation schemes [3][4]. These two concepts require a tradeoff for the transmission rang so that the range should be short enough to save energy and avoid high costs of data transmission and long enough to ensure no splitting of the network and achieve high data throughput. 
In previous studies [6][7], the authors proposed algorithms which assign minimum transmission range with ensuring network connectivity, but they require global information about node locations which is difficult to achieve in WSNs. Furthermore, in [8], a Local Minimum Spanning Tree (LMST) algorithm was proposed with less demanding solution. The authors of [1] analyze end-to-end connectivity with respect to deployment density of network's nodes and provide an analytical solution. Inspired with the work of [1], we modified their algorithm by using a simpler mathematical approach for computing the average anguler deviation $(\overline{\boldsymbol{\theta}})$ and the next hop distance $\left(\boldsymbol{d}_{\text {Next }}\right)$. The modified approach reduces $\mathrm{CH}$-to-CH transmission ranges and provides more conserving $\mathrm{CH}$ transmission power while maintaining high probability of end-to-end connectivity to the gateway.

The remainder of this paper is organized as follows. Section 2 describes the used mathematical approach for assigning the minimum transmission range. Section 3 provides numerical results and comparison of the original approach in [1]. Finally, Section 4 concludes the paper.

\section{Minimum Transmission RAnge Computation}

We follow the approach in [1] to compute the minimum transmission range by increasing $R$ until obtaining 95\% end-to-end connectivity probability (Prob) for a $\mathrm{CH}$ node located at a distance $d$ from the gateway as shown in Algorithm 1 . Where $R_{0}$ is the initial value of $R$ and $\Delta R$ is the range increment.

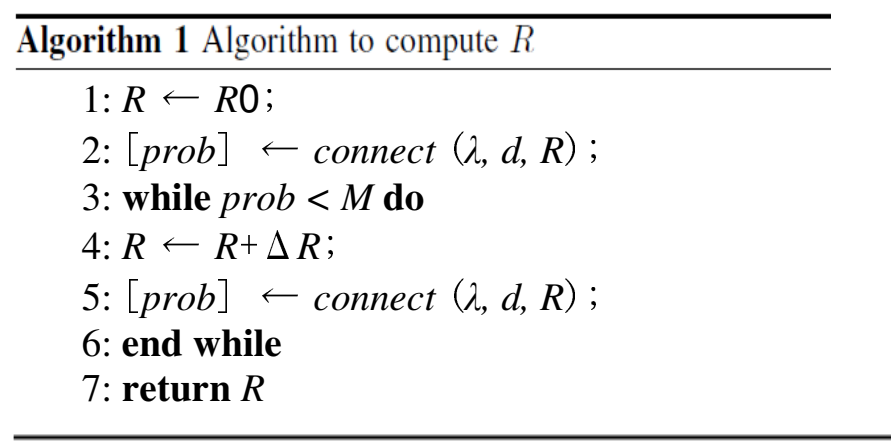

The procedure (connect) returns prob, which is obtained by using algorithm 2 . Hence, the probability of end-to-end connectivity to the gateway for a given $\mathrm{CH}$ node density $\lambda$, and a communication range $\mathrm{R}$ of $\mathrm{CH}$-to- $\mathrm{CH}$ is calculated by the procedure connect as shown below.

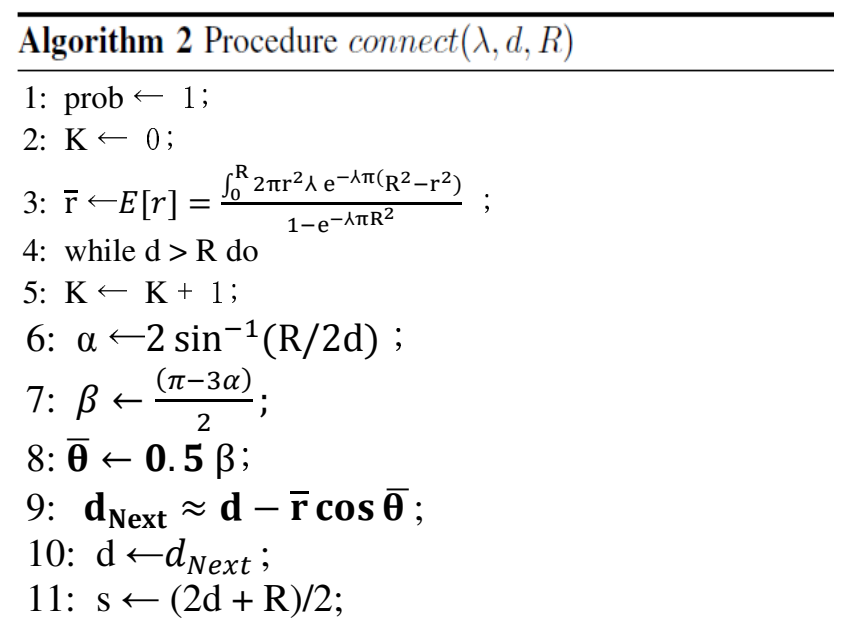


12: $\mathrm{a} \leftarrow \sqrt{\mathrm{s}(\mathrm{s}-\mathrm{d})^{2}(\mathrm{~s}-\mathrm{R})}$

13: Area $_{1} \leftarrow \mathrm{d}^{2} \alpha / 2-\mathrm{a}$;

14: Area $_{2}=\frac{\mathrm{R}^{2} \beta}{2}$;

15: Area $\left(R_{\text {Next }}\right)=2\left(\right.$ Area $_{1}+$ Area $\left._{2}\right)$;

16: if $\mathrm{K}==1$ then

17: prob $\leftarrow\left(1-\mathrm{e}^{-\lambda \text { Area }\left(R_{\text {Next }}\right)}\right)$ prob ;

18: else

19: $\left(R_{\text {new }}\right) \leftarrow((2 \mathrm{~K}-3) \overline{\mathrm{r}}+2 \mathrm{R}) \overline{\mathrm{r}} \bar{\theta}$

20: prob $\leftarrow\left(1-\mathrm{e}^{-\lambda \text { Area }\left(R_{\text {new }}\right)}\right)$ prob ;

21: end if

22: end while

23: if $\mathrm{d}>0$ then

24: $\mathrm{K} \leftarrow \mathrm{K}+1$;

25: end if

26: return prob

The idea of algorithm 2 in [1] is to get the shortest hop distance between the next hop node of $\boldsymbol{A}$ (denoted by $A_{N e x t}$ ) and the gateway $\boldsymbol{B}$. where, node $\boldsymbol{A}$ is originally at a distance $\boldsymbol{d}$ from node $\boldsymbol{B}$, as shown in Figure 1(a). Hence, Region $A$ is the intersection of $\quad B^{\prime}$ 's circular $\operatorname{arc}$ of radius $d\left(X^{\prime} Y^{\prime}\right)$ with $A$ 's circular range. Similarly, Region $B$ is the intersection of $A$ 's circular $\operatorname{arc}$ of radius $d(X Y)$ with $B$ 's circular range.

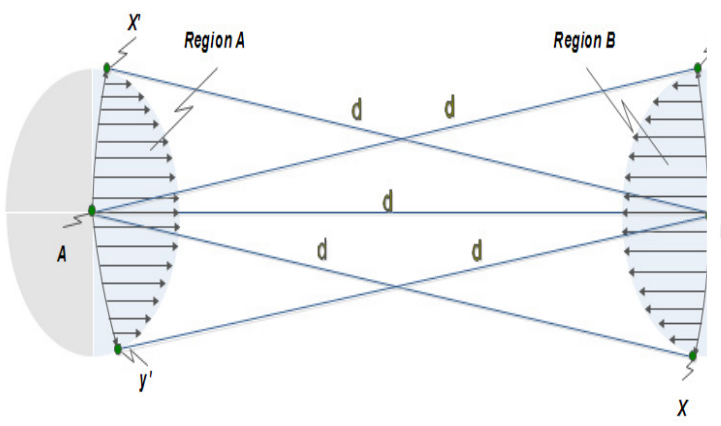

a) Region $A$ and Region $B$

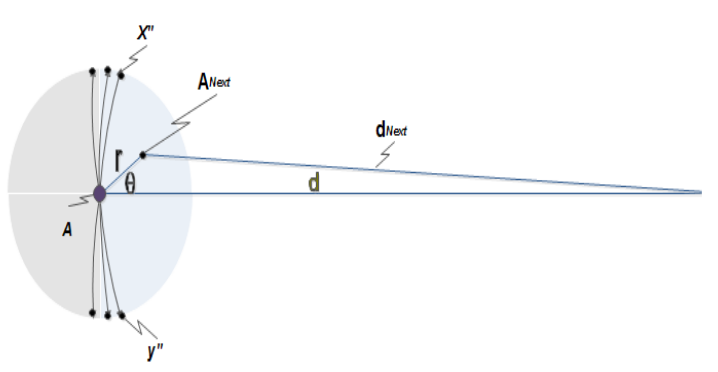

c) Next hop $\boldsymbol{A}_{\text {Next }}$ and new distance

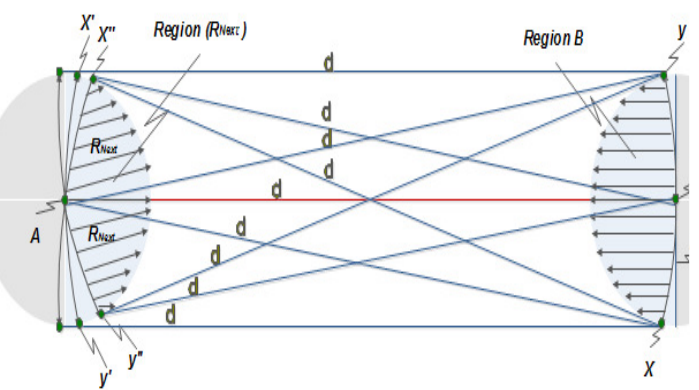

b) Region $R_{\text {Next }}\left(\right.$ Area $\left.\left(R_{\text {Next }}\right)\right)$

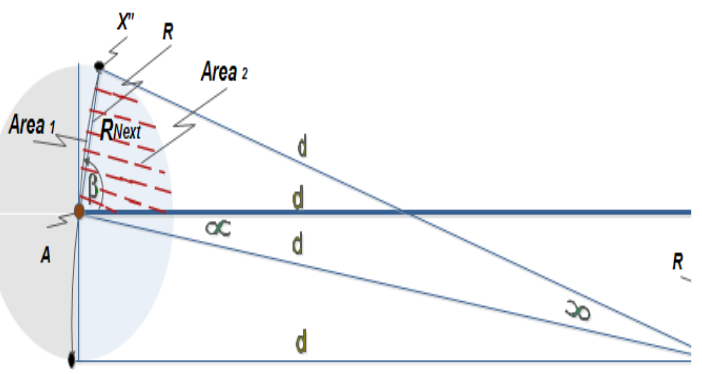

d) Area $_{1}$ and Area $_{2}$

$$
d_{\text {Next }}
$$

Fig.1: Locating the next hop node towards the gateway. [1] 
Figure 1(b), illustrates the determination of Region $R_{\text {Next }}$ (denoted as Area $\left(R_{\text {Next }}\right)$, in line 15 of algorithm2) within region $A$, to ensure that the distance $d_{\text {Next }}$ (which is the distance between $A$ 's next hop and $B$ 's previous hop) is always less than the distance $d$. Hence, the Region $R_{\text {Next }}$ is created by the intersection of the circular arcs of radius $d$ form points $X$ and $Y$ with region $A$ (in Fig. 1(a)) at points $X^{\prime \prime}$ and $Y^{\prime \prime}$ respectively.

Therefore, when we randomly select next hop for node $A$ in Region $R_{\text {Next }}$, it will have a new distance $\left(d_{N e x t}\right)$ to gateway $B$ less than the previous distance $d$. This means that the distance to gateway $B\left(d_{\text {Next }}\right)$ monotonously decreases with increasing hop count $K$ (see line 5 of algorithm 2) to node $A$ and re-find a new value $d_{N e x t}<d$ (line 10) until $d<R$. This is clear from the inner loop of algorithm 2 (line 16 to line21). Also this loop updates the probability (Prob) of end-to-end connectivity over $K$ hops at each iteration.

Hence, we can obtain the probability to find at least one node in Area $\left(R_{\text {Next }}\right)$ to be the next first hop of $A\left(A_{\text {Next }}\right)$ as $\left(\mathbf{1}-\mathbf{e}^{-\lambda \operatorname{Area}\left(\boldsymbol{R}_{\text {Next }}\right)}\right)$, (line 17 in algorithm 2$)$. Area $\left(R_{\text {Next }}\right)$ is represented by dashed lines in Figure 1(b), (line 15 in algorithm 2), and $R_{\text {Next }}$ is represented by dashed lines in Figure 1(d). It should be noted that:

$$
\begin{aligned}
& R_{\text {Next }}=\text { Area }_{1}+\text { Area }_{2} \text {, and Area }\left(R_{\text {Next }}\right)=2 * R_{\text {Next }} \\
& \text { Area }\left(R_{\text {Next }}\right)=2\left[\text { Area }_{1}+\text { Area }_{2}\right] \\
& \text { Area }_{2}=\frac{R^{2} \beta}{2} \\
& \text { Area }_{1}=d^{2} \alpha / 2-a \\
& \text { and, } a=\sqrt{s(s-d)^{2}(s-R)}
\end{aligned}
$$

where $(a)$ is the area of triangle $\mathrm{AX}^{/ /} \mathrm{X}$, and $(s)$ is the half circumference of the triangle, (lines 1115 of algorithm 2).

As shown in Figure 2, the area Area $R_{\text {new }}$ has new next hops for nodes of hop number $i$. where this area is presented by a simple geometrical calculation as $\operatorname{Area}\left(R_{\text {new }}\right) \leftarrow((2 \mathrm{~K}-3) \overline{\mathrm{r}}+2 \mathrm{R}) \overline{\mathrm{r}} \bar{\theta}$ (see line19 of algorithm 2). Then, we consider the probability to find at least one node in Area Rnew to update prob (see line 20). [1][9]

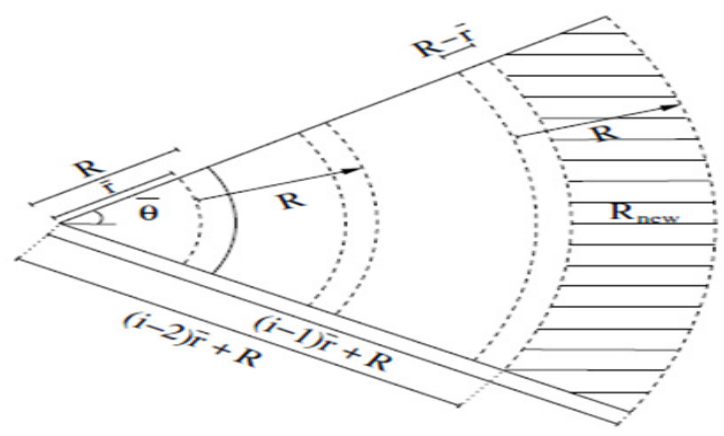

Fig. 2: Search area for next hop nodes. [1] 
For an $X \times X$ network, where ( $X=1000 \mathrm{~m}$ ), the derived terms used in algorithm 2 can be accurate enough when the range $R$ is close to the value of distance $(d)$ which is selected by Monte Carlo simulations to obtain the minimal range $R$ that gives a connectivity of at least $95 \%$, for different values of node density $\sigma$. Therefore, the best estimation is obtained when choosing the $\mathrm{CH}$ that is $X / 2(d=500 \mathrm{~m})$ away from the gateway for all density values [1]. Note that $\lambda=p \sigma$, where $p$ is the $\mathrm{CH}$ selection probability.

\subsection{Computation of $d_{\text {Next }}[1]$}

In [1], and as shown in Figure 1(c), $d_{N e x t}$ is approximated by using the following formula:

$$
d_{\text {Next }} \approx \sqrt{\bar{r}^{2}+d^{2}-2 d \bar{r} \cos \bar{\theta}}
$$

Where $\bar{r}$ is the expected value of the minimum radial distance, $0<r \leq R$, which is computed by using line 3; and $\bar{\theta}$ is the average angular deviation, $0 \leq \theta \leq \beta$, which is computed in [1] by using the following formula:

$$
\bar{\theta}=\frac{\left.\int_{0}^{\beta} \theta e^{-2 \wedge\left[\frac{\theta \bar{r}^{2}-\bar{r}^{2}}{2} \sin (2 \theta)\right.}\right] d \theta}{\int_{0}^{\beta} e^{-2 \curlywedge\left[\frac{\theta \bar{r}^{2}-\frac{\bar{r}^{2}}{2} \sin (2 \theta)}{2}\right]} d \theta}
$$

\subsection{Computation of $d_{\text {Next }}$ (our approach)}

As shown in Figure 3, the idea is that the component of $\bar{r}$ on the straight line $A B$ is $(\bar{r} \cos \bar{\theta})$. Therefore we can also approximate $d_{\text {Next }}$ as:

$$
d_{N e x t} \approx d-\bar{r} \cos \bar{\theta}
$$

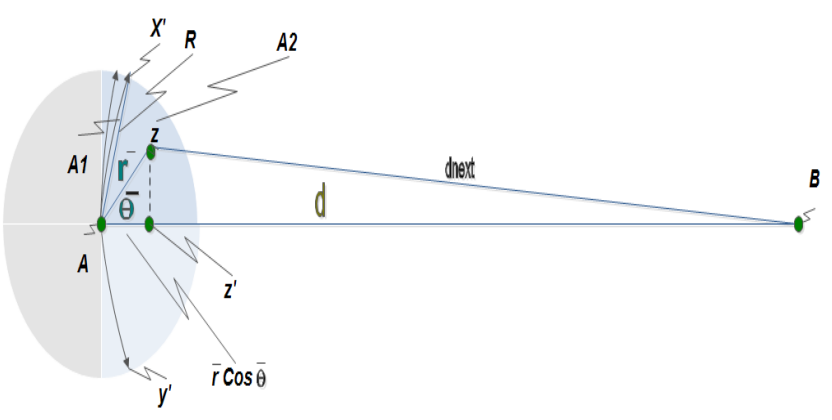

Fig.3: $A_{\text {Next }}$ and new distance $d_{\text {Next }}$ (our approach) 


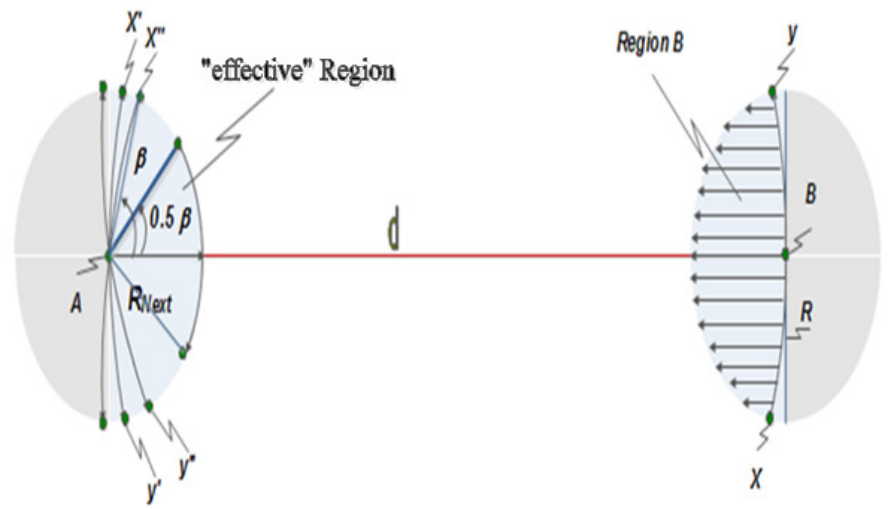

Fig. 4: Effective Region

Moreover, as shown in Figure 4, if we choose $A_{\text {Next }}$ to be in the indicated "effective" region of Region $R_{N e x t}$, then we can obtain the highest transmission range for a given transmission power. Hence, the average angular deviation $(\bar{\theta})$ which was computed by equation (3) in [1] can be approximated as:

$$
\bar{\theta} \approx 0.5 \beta
$$

Then we can find the transmission power by using the following formula [2]:

$$
P_{A B}=R^{2}
$$

Where $P_{A B}$, is the minimum required transmission power from node A to getaway $\mathrm{B}$, and $R$ is the transmission range in $(\mathrm{m})$. Therefore we can find the Power saving percentage for minimum transmission ranges as follows:

$$
\text { Power saving } \%=\left(1-\frac{P_{\text {New }}}{P_{\text {old }}}\right) \times 100 \%
$$

Where, $P_{\text {old }}$ is the transmission power calculated by using the approach of [1], and $P_{N e w}$ is the transmission power calculated by using our approach.

\section{Performance Evaluation}

Numerical analysis was performed to find the end-to-end connectivity probability as a function of the minimum transmission range $R$. The analysis were done for various node density values $(\sigma$ $=3 \times 10^{-3}, 4 \times 10^{-3}, 5 \times 10^{-3}$, and $\left.6 \times 10^{-3}\right)$. The $\mathrm{CH}$ selection probability $p$ is taken as 0.1 , corresponding to $\mathrm{CH}$ density values $\left(\lambda=3 \times 10^{-4}, 4 \times 10^{-4}, 5 \times 10^{-4}\right.$, and $\left.6 \times 10^{-4}\right)$, whereas $R_{O}=$ $10 \mathrm{~m}$ and $\Delta R=1 \mathrm{~m}$. Figure 5 and Figure 6 show the connectivity probability as a function of the transmission range for the approach in [1] and our approach respectively. As expected, in both figures, end-to-end connectivity probability increases as the range $R$ increases, and also it increases as the node density increases. This is because when the range or node density increases, the chance of $\mathrm{CHs}$ to find next hop is more, and hence, communication is more assured.

Since the objective is to achieve a high end-to-end connectivity probability with a minimum transmission range, we summarized the comparison of both Figures at $95 \%$ connectivity probability for the various node density values as shown in Table 1. It is clear that our approach outperforms the approach of [1] by $19.40-28.56 \%$ power saving since we succeeded to assign lower minimum transmission ranges. 
International Journal of Computer Networks \& Communications (IJCNC) Vol.8, No.2, March 2016

Table1. Power saving \% Comparison for 95\% Connectivity Prob.

\begin{tabular}{|c|c|c|c|}
\hline $\begin{array}{c}\text { Node } \\
\text { density } \\
\sigma\end{array}$ & $\begin{array}{r}\text { Range } R \text { for } \\
\text { approach [1] } \\
(\mathrm{m})\end{array}$ & $\begin{array}{r}\text { Range R for } \\
\text { our approach } \\
(\mathrm{m})\end{array}$ & $\begin{array}{r}\text { Power } \\
\text { saving\% }\end{array}$ \\
\hline 0.003 & 98 & 88 & 19.40 \\
\hline 0.004 & 84 & 71 & 28.56 \\
\hline 0.005 & 74 & 65 & 22.85 \\
\hline 0.006 & 67 & 59 & 22.46 \\
\hline
\end{tabular}

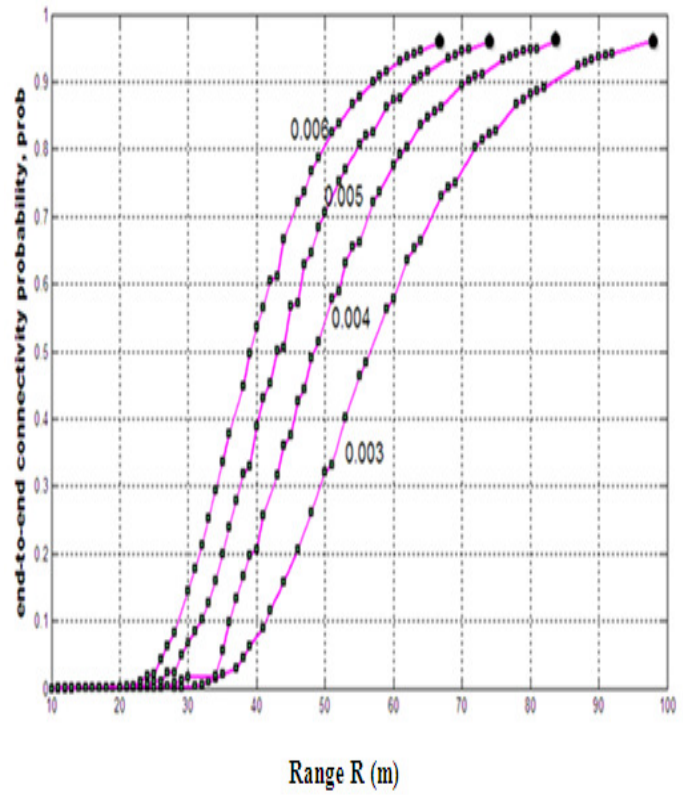

Fig.5. Connectivity Probability versus $R$ for the approach of [1]

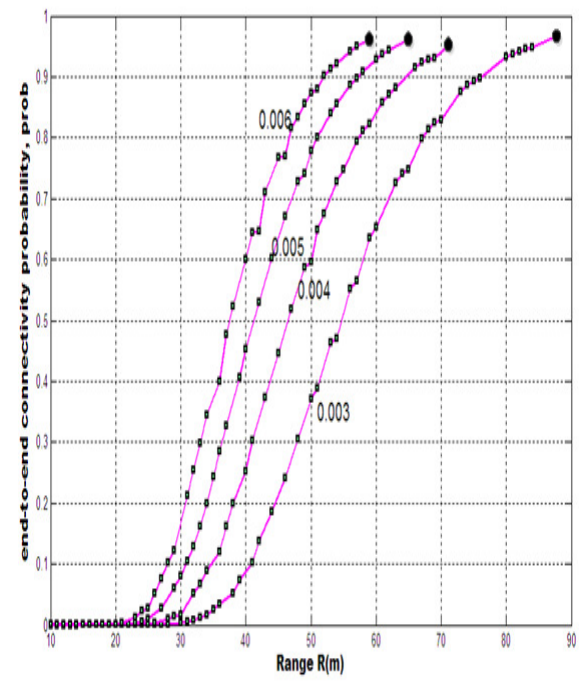

Fig.6. Connectivity Probability versus R for our approach 


\section{Conclusions}

Transmission range assignment in WSNs is an important issue which affects the transmission power and connectivity of the nodes. Therefore, the ultimate goal is to maintain high connectivity probability with minimum transmission range so that energy conservation and data delivery are both achieved. in this paper, we followed a similar analytical approach given in [1] to assign the minimum transmission range for $\mathrm{CH}$-to- $\mathrm{CH}$ communication with an acceptable connectivity probability.

Our approach differs from the approach given in [1] in two different aspects: 1) we used a simpler mathematical model; 2) we maintained the same connectivity with smaller transmission ranges, which means less power consumption and hence longer life time of the nodes. In summary, the numerical results demonstrate that our proposed approach is more effective in prolonging the network lifetime.

\section{ACKNOWLEDGEMENTS}

We sincerely thank our colleague Mr. Kilan M. Hussein for his help during the programming process.

\section{REFERENCES}

[1] Serdar Vural, Pirabakaran Navaratnam, and Rahim Tafazolli "Transmission Range Assignment for Backbone Connectivity in Clustered Wireless Networks", IEEE Trans. Wireless Commun., Vol. 2, No. 1, pp. 46-49, Feb. 2013.

[2] Abolfazl Akbari, Neda Beikmahdavi, "Coverage and Clustering Guarded for Wireless Sensor Network", Int. J. Latest Trends Computing Vol. 2, No. 3, pp. 465-472, Sept., 2011.

[3] G. Chen, C. Li, M. Ye, and J. Wu, "An unequal cluster-based routing protocol in wireless sensor networks”, Springer Wireless Networks, pp. 193-207, April 2007.

[4] D. Wei, Y. Jin, S. Vural, and R. Tafazolli, "An energy-efficient clustering solution for wireless sensor networks,” IEEE Trans. Wireless Commun., Vol. 10, No. 11, pp. 1-11, September 2011.

[5] O. Younis and S. Fahmy, "HEED: a hybrid, energy-efficient, distributed clustering approach for ad hoc sensor networks," IEEE Trans. Mobile Comput., Vol. 3, No. 9, pp. 366-379, Dec. 2004.

[6] Q. Dai and J. Wu, "Computation of minimal uniform transmission range in ad hoc wireless networks,” Springer J. Cluster Comput., Vol. 8, No.2-3, pp. 127-133, 2005.

[7] R.Ramanathan and R. Hain, "Topology control of multihop wireless networks using transmit power adjustment," IEEE Info com., Vol. 2, pp. 404-413, 2000.

[8] F. J. O. Mart'inez, I. Stojmenovi'c, F. G. Nocetti, and J. S. Gonz'alez, "Finding minimum transmission radii for preserving connectivity and constructing minimal spanning trees in ad hoc and sensor networks," Elsevier J. Parallel and Distributed Comput., pp. 132-141, 2005.

[9] S. Vural and E. Ekici, "On multihop distances in wireless sensor networks with random node locations,” IEEE Trans. Mobile Comput., vol. 9, no. 4, pp. 540-552, Apr. 2010. 


\section{AUTHORS}

Muhammed SALAMAH received the B.S., M.S., and PhD. degrees in Electrical and Electronics Engineering from Middle East Technical University in 1988, 1990, and 1995, respectively. From 1988 to 1996, he worked as a Research Assistant and Lecturer in METU. In 1995, he worked as a visiting researcher in the Networks Department of Ecole Nationale Superieure des Telecommunications in Paris, France. Since 1996, he has been with Department of Computer Engineering at Eastern Mediterranean University, TRNC, Turkey, where he is an Assoc. Professor. He served as the Assistant Chairman of the Computer Engineering Department of EMU from 1997 to 2004; the chair of the Student Project and Design Center in EMU from 2005 to 2008; Rectorate Coordinator for Student Affairs and Informatics from 2008 to 2010; head of the Computer Engineering Department of EMU from 2010 to 2013, and since 2013 he has been serving as the Rector's Advisor for Middle East in EMU. His current research interests are computer networks and wireless networks with emphasis on resource allocation and management, performance evaluation, multiprocessing, and hardware-oriented algorithms.

Abd Ali Hussein was born in Diyala, Iraq, in 1970. He received the B.S. degree in electrical engineering in 1993. He is Employee of the University of Diyala, now he studies M.S. degrees in Computer engineering department of Eastern Mediterranean University (EMU) University

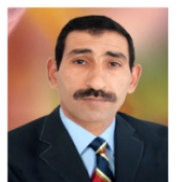

